\title{
Eimeria intricata Spiegel, 1925 em ovinos de Mossoró, Rio Grande do Norte, Brasil*
}

\author{
Eimeria intricata Spiegel, 1925 in sheep Mossoro, \\ Rio Grande do Norte, Brazil
}

\author{
Josivania Soares Pereira, ${ }^{* *}$ Guilherme Moniz Sodré Lopes Teixeira, ${ }^{* *}$ Arickson Wesley da Silva Pereira, ${ }^{* *}$ \\ Zuliete Aliona Araújo de Souza Fonseca, ${ }^{* * *}$ Kaliane Alessandra Rodrigues de Paiva, ${ }^{* *}$ \\ Iris da Silva Marques, ${ }^{* *}$ Sílvia Maria Mendes Ahid**
}

\begin{abstract}
Resumo
Objetivou-se identificar através da morfometria, espécimes de Eimeria em ovinos. Realizou-se oocistograma (OOPG) em 50 ovinos da raça Dorper de Mossoró, RN. As amostras fecais positivas no OOPG foram submetidas à esporulação em solução aquosa de bicromato de potássio $2,5 \%$ por sete dias, sob temperatura ambiente $\left(\cong 27^{\circ} \mathrm{C}\right)$. Foi feita identificação de 100 oocistos selecionados aleatoriamente no exame de microscopia óptica (objetiva de $40 \mathrm{X}$, fator de correção 0,333 ). Os dados foram expressos em média, desvio padrão, valores mínimos e máximos, calculados pelo programa estatístico SPSS (Statistical Package for the Social Sciences) 21.0. Os coccídeos, objeto deste trabalho, classificados em Eimeria intricata Spiegel, 1925 apresentaram: oocisto com média de comprimento $50,83 \mu \mathrm{m}(43,29-53,28 \mu \mathrm{m})$; largura média $36,18 \mu \mathrm{m}(33,30-39,96 \mu \mathrm{m})$ e índice morfométrico

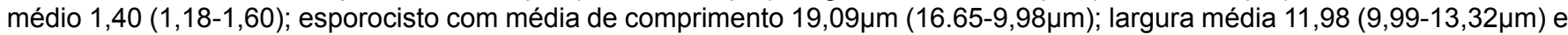
índice morfométrico médio 1,60 $\mu \mathrm{m}(1,50-2,0 \mu \mathrm{m})$. Este registro amplia o conhecimento da ocorrência de $E$ intricata em mais uma localidade do Nordeste brasileiro e auxilia a reconhecer que a existência da mesma nos rebanhos ovinos do Rio Grande do Norte pode não desencadear quadros patogênicos, mas indica falhas no manejo dos animais.
\end{abstract}

Palavras-chave: Eimeriose, esporocisto, Oocisto.

\begin{abstract}
The objective of this study was to identify morphometry, Eimeria specimens of Dorper sheep of Mossoró, RN. Oocyst (OOPG) was performed on 50 sheep. Positive faecal samples in the OOPG were submitted to sporulation in aqueous solution of $2.5 \%$ potassium dichromate for seven days, at room temperature $\left(\cong 27^{\circ} \mathrm{C}\right)$. Identification of 100 randomly selected oocysts was performed on the optical microscopy (objective 40X, correction factor 0.333). Data were expressed as mean, standard deviation, minimum and maximum values, calculated by the statistical program SPSS (Statistical Package for the Social Sciences) 21.0. The

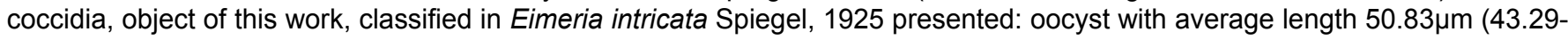
$53.28 \mu \mathrm{m})$; Mean width $36.18 \mu \mathrm{m}(33.30-39.96 \mu \mathrm{m})$ and mean morphometric index 1.40 (1.18-1.60); Sporocyst with a mean length of $19.09 \mu \mathrm{m}(16.65-9.98 \mu \mathrm{m})$; Mean width $11.98(9.99-13.32 \mu \mathrm{m})$ and mean morphometric index 1.60 $\mu \mathrm{m}(1.50-2.0 \mu \mathrm{m})$. This record amplifies the knowledge of the occurrence of $E$. intricata in another locality of the Northeast of Brazil and helps to recognize that the existence of the same in the sheep flocks of Rio Grande do Norte may not trigger pathogenic conditions, but indicates failures in the management of the animals.
\end{abstract}

Keywords: eimeriosis, oocyst, sporocyst.

\section{Introdução}

As parasitoses gastrintestinais assumem relevância, considerando-se as elevadas perdas econômicas decorrentes da baixa produtividade dos animais adultos, da elevada mortalidade e do atraso no desenvolvimento corporal dos jovens (Ahid et al., 2008). Dentre as enfermidades que acometem os ovinos, às eimerioses contribuem para aumentar a mortalidade e constituir um obstáculo ao melhoramento dos índices da produção animal, manifestando- se por transtornos intestinais com diarreia aquosa e hemorrágica, além de má condição corporal (Grilo e Carvalho, 2014).

No Rio Grande do Norte, o parasitismo por Eimeria sp. desencadeia em ovinos infecções subclínicas que limitam a produtividade dos rebanhos. Neste estado, oocistos deste protozoário foram registrados para região central, Lajes, por Souza et al. (2012) e para região oeste, por Ahid et al. (2008). Espécies de Eimeria identificadas por técnicas morfométricas sp.

*Recebido em 14 de fevereiro de 2017 e aceito em 12 de julho de 2017

**Universidade Federal Rural do Semi-Árido. Centro de Ciências Biológicas e da Saúde. Av. Francisco Mota, 572 - Bairro Costa e Silva, Mossoró RN, 59.625-900. Autor para correspondência: josigej@ufersa.edu.br

***Faculdade de Enfermagem e de Medicina Nova Esperança, Av. Presidente Dutra, 701 - Alto de São Manoel, Mossoró - RN, $59628-000$. 
foram notificadas na região Leste, Macaíba, por Andrade Júnior et al. (2012). Dentre as espécies foram identificadas Eimeria ovina, Eimeria ahsata, Eimeria crandallis, Eimeria faurei; Eimeria intrincata, Eimeria ovinoidalis, Eimeria pallida, Eimeria parva e Eimeria granulosa. Considerando a cidade de Mossoró, região Oeste do RN, o registro de espécies de Eimeria foi feito apenas por Ahid et al. (2009) que identificaram E. ovina, E. ovinoidallis, E. parva, E. faurei, E. granulosa, E. ahsata, Eimeria crandallis e Eimeria caprovina.

O conhecimento de espécies de Eimeria que acomete ovinos no RN, faz-se necessário para determinação de espécies patogênicas, bem como implantação adequada de medidas de controle e formulações de tratamento a estes protozoários. Objetivou-se identificar através da morfometria, espécimes de Eimeria em ovinos Dorper de criação semiextensiva provenientes de Mossoró, RN.

\section{Material e métodos}

Realizou-se oocistograma (OOPG) em 50 ovinos. Todos os procedimentos de manipulação e as normas de ética com o uso de animais foram executados conforme as recomendações propostas pela Comissão de Ética no uso de animais em pesquisa da UFERSA.

As amostras fecais foram coletadas diretamente da ampola retal e processadas no UFERSA. Quando positivas no OOPG, as amostras foram submetidas à esporulação em solução aquosa de bicromato de potássio $2,5 \%$ por sete dias, sob temperatura ambiente $\left(\cong 27^{\circ} \mathrm{C}\right)$ e segundo técnica descrita por Vieira et al. (1999) e Fonseca et al. (2012).

Após esporulação, os oocistos foram separados pela técnica de centrifugoflutuação em solução saturada de sacarose. Os mesmos foram concentrados segundo Menezes e Lopes (1995) e identificados com base nas descrições e medições morfométricas, conforme descrito por Levine (1982).

\section{Resultados e discussão}

Todas as amostras fecais obtidas dos animais apresentaram parasitismo para coccídeos de Eimeria intricata Spiegel, 1925. Foram medidos 100 oocistos (microscopia óptica, objetiva de 40X, fator de correção 0,333). Estes apresentaram: oocisto com média de comprimento $50,83 \mu \mathrm{m}(43,29-53,28 \mu \mathrm{m})$; largura média $36,18 \mu \mathrm{m}(33,30-39,96 \mu \mathrm{m})$ e índice morfométrico médio 1,40
(1,18-1,60); esporocisto com média de comprimento $19,09 \mu \mathrm{m}$ $(16.65-9,98 \mu \mathrm{m})$; largura média $11,98(9,99-13,32 \mu \mathrm{m})$ e índice morfométrico médio $1,60 \mu \mathrm{m}(1,50-2,0 \mu \mathrm{m})$ (Figura 1). Souza et al. (2015) encontraram também em condições semiáridas da região Nordeste $E$. intricata acometendo ovinos com índice morfométrico para os oocistos exatamente igual ao presente trabalho $(\mathrm{IM}=1,4 \mu \mathrm{m})$.

Segundo Kareem e Yücel (2015), E. intricata não é uma espécie que comumente é descrita acometendo ovinos nos diferentes países do mundo. Porém este coccídeo já foi relatado para ovinos na Polônia (Antoszek e Balicka-Ramisz, 2009); no Irã (Yakhchali e Rezaei, 2010); na China (Wang et al., 2010); na Alemanha (Dittmar et al., 2010). No Brasil, foi notificada na região Sudeste, São Paulo (Amarante e Barbosa, 1992) e no Rio de Janeiro (Hassum et al., 2005); no Norte, Tocantins (Almeida et al., 2013); no Sul, Paraná (Lopes et al., 2013); e no Nordeste, Pernambuco (Tembue et al., 2009); Bahia (Souza et al., 2015). No Rio Grande do Norte, E. intricata foi notificada em ovinos no município de Lajes (Silva, 2009), em Angicos (Silva et al., 2011) e em Macaíba, por Andrade Júnior et al. (2012).

No que diz respeito a patogenicidades aos ovinos, das espécies de Eimeria descritas para o RN, a mais preocupante é E. ovinoidallis, por ocasionar anorexia, perda de peso, diarreia intensa e morte dos animais. De efeito moderado, há E. crandallis, E. bakuensis e E. parva. Já E. intricata, registrada no RN por Andrade Júnior et al. (2012) e através do presente trabalho notificada pela primeira vez na cidade de Mossoró, apresenta patogenicidade desconhecida ao acometer ovinos.

Segundo Vercruysse (1982), Chhabra e Pandey (1991) e Tembue et al. (2009), E. intricata acomete, embora em percentuais baixos e suficientes para não desencadear quadro patogênico, não apenas os ovinos, mas também caprinos. Nestes animais, observa-se que E. intricata é um coccídeo que apesar de presente, não representa um grave problema para estes organismos, pois os mesmos podem não ser os responsáveis pelas fortes diarreias sanguinolentas e perdas nos rebanhos. Vale ressaltar que apesar de $E$. intricata ser relatada como uma espécie de patogenicidade desconhecida, além de outras espécies registradas como protozoários de baixa patogenicidade, a notificação da ocorrência de todas elas no Brasil e no mundo auxilia na ampliação do conhecimento das espécies que acometem ovinos e que a presença das mesmas juntamente com outras espécies nos rebanhos pode ser um indicativo de falhas no manejo dos animais.

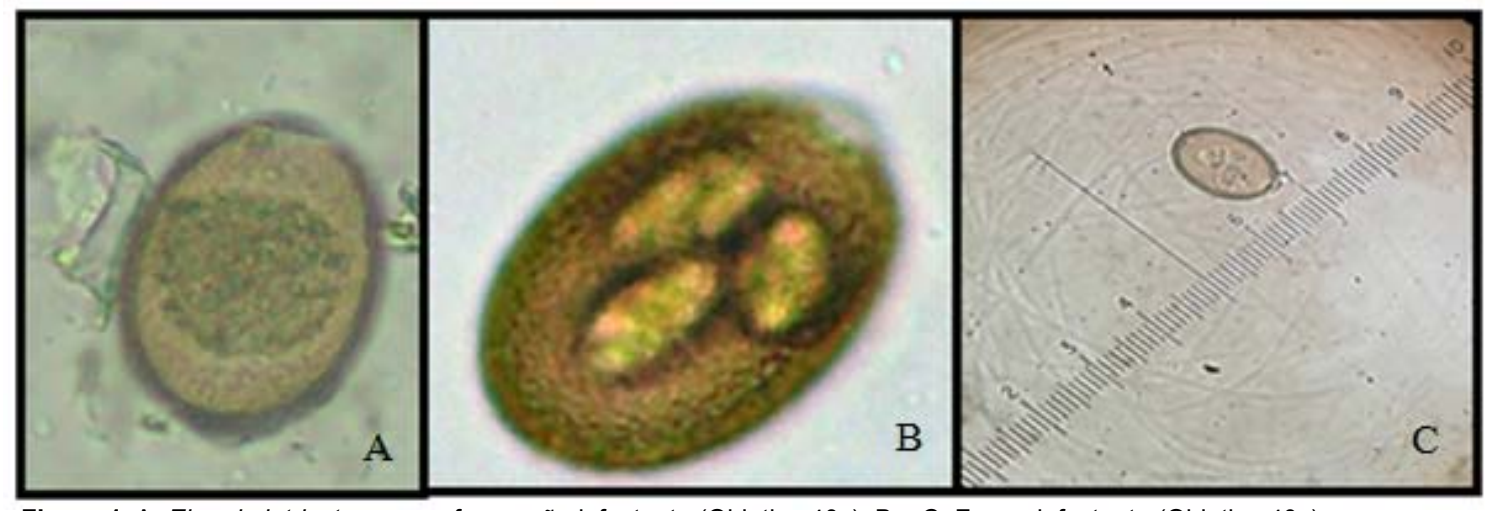

Figura 1: A: Eimeria intricata em sua forma não infectante (Objetiva 40x); B e C: Forma infectante (Objetiva 40x) 


\section{Referências}

AHID, S.M.M.; SUASSUNA, A.C.D.; MAIA, M.B.; COSTA, V.M.M.; SOARES, H.S. Parasitos gastrintestinais em caprinos e ovinos da região oeste do rio grande do norte, Brasil. Ciência Animal Brasileira, v. 9, n. 1, 2008).

AHID, S.M.M.; MEDEIROS, V.M.C.; BEZERRA, A.C..D.S.; MAIA, M.B.; XAVIER, V.M.; VIEIRA, L.S. Espécies do gênero Eimeria Schneider, 1875 (Apicomplexa: Eimeriidae) em pequenos ruminantes na mesorregião oeste do estado do Rio Grande do Norte, Brasil. Ciência Animal Brasileira, v.10, n. 3, p. 984-989, 2009.

ALMEIDA, J.D.M.; MACHADO, A.S.; TOLEDO, G.A.; COSTA, A.M.; FREITAS, WLC 2, FREITAS, F.L.C. Infection due to Eimeria spp. in sheep in the municipality of Colinas, state of Tocantins. Revista de Medicina Veterinária, v.7, n.4, p.33-36, 2013.

AMARANTE, A.F.; BARBOSA, M.A. Species of coccidia occurring in lambs in São Paulo State, Brazil. Veterinary Parasitology, v. 41, p.189-193, 1992.

ANDRADE JÚNIOR, A.L.F.; SILVA, P.C.; AGUIAR, E.M.; SANTOS, F.G.A. Use of coccidiostat in mineral salt and study on ovine eimeriosis. Revista Brasileira de Parasitologia Veterinária, v. 21, n. 1, p. 16-21, 2012.

ANTOSZEK, J.; BALICKA-RAMISZ, A. Occurrence of Eimeria protozoa in lambs in Western Pomerania, Poland. Wiad Parazytol, v. 55, n. 1, p. 35-38, 2009.

CHHABRA, R.C.; PANDEY, V.S. Coccidia of goats in Zimbabwe. Veterinary Parasitolology, v. 39, p. 199-205, 1991.

DITTMAR , K.; MUNDT, H.C.; GRZONKA, E.; DAUGSCHIES, A.; BANGOURA, B. Ovine coccidiosis in housed lambs in SaxonyAnhalt (central Germany). Berliner und Münchener tierärztliche Wochenschrift, v.123, p.49-57, 2010.

FONSECA, Z.A.A.S.; AVELINO, D.B.; BEZERRA, A.C.A; MARQUES, A.S.C.; PEREIRA, J.S.; COELHO, W.A.C.; VIEIRA, L.S.; AHID, S.M.M. Espécies de Eimeria sp. em matrizes caprinas leiteiras no município de Afonso Bezerra-RN. Acta Veterinaria Brasilica, v. 6, n. 2, p.131-135, 2012.

GRILO, M.L.; CARVALHO, L.M. Coccidiose em ruminantes pequenos agentes e grandes problemas nas diarreias parasitárias. Revista de Medicina Veterinária, v.1, p. 34-48, 2014.

HASSUM, I.C.; MENEZES, R.C.A.A. infecção natural por espécies do gênero Eimeria em pequenos ruminantes criados em dois municípios do estado do Rio de Janeiro. Revista Brasileira de Parasitologia Veterinária, v.14, n. 3, p. 95-100, 2005.

KAREEM, S.I.; YÜCEI, Ş.Y. Prevalence of Eimeria Species in Sheep in Sulaimaniya Province, Iraq. Journal of Entomology and Zoology Studies, v. 3, n. 4, p. 317-322, 2015.

LEVINE, N. D. Taxonomy and life of cycles of coccidian. In:LONG, L. P. The Biology of the coccidian. Baltimore, University Park

Press. 1-33 p. 1982
LOPES, W.D.Z; BORGES, F.A.; FAIOLLA, T.P.; ANTUNES, L.T.; BORGES, D.G.L.; RODRIGUEZ, F.S. FERRARO, G.; TEIXEIRA, W.F.; MACIEL, W.G.; FELIPPELLI, G. COSTA, A.J.; PEREIRA, V.; MARTINEZ, A.C. Eimeria species in young and adult sheep raised under intensive and / or semi-intensive systems of a herd from Umuarama city, Parana State, Brazil. Ciência Rural, v.43, n .11, p. 2031-2036, 2013.

MENEZES, R.C.A.A.; LOPES, C.W.G. Epizootiologia da Eimeria arloingi em caprinos na microrregião serrana fluminense, Rio de Janeiro, Brasil. Revista Universidade Rural, v. 17, n. 12, p. 5-12, 1995.

SILVA, R.M. Infecção natural por Eimeria spp., Cryptosporidium spp. e Giardia duodenalis em cordeiros da raça mestiça Santa Inês, na região semi-árida do Estado do Rio Grande do Norte. Dissertação de mestrado em Parasitologia, Belo Horizonte, 2009. $94 \mathrm{f}$.

SILVA, R.M.; FACURY-FILHO, E.J.; SOUZA, M.F.; RIBEIRO, M.F.B. Natural infection by Eimeria spp. in a cohort of lambs raised extensively in Northeast Brazil. Revista Brasileira de Parasitologia Veterinária, v. 20, n. 2, p. 134-139, 2011.

SOUZA, L.E.B.; CRUZ, J.F.; NETO, M.R.T.; ALBUQUERQUE, G.R.; MELO, A.D.B.; TAPIA, D.M.T. Epidemiology of Eimeria infections in sheep raised extensively in a semiarid region of Brazil. Brazilian Journal Veteterinary Parasitology, v. 24, n. 4, p. 410-415, 2015.

SOUZA, M.F.; PIMENTEL-NETO, M.; SILVA, R.M.; FARIAS, A.C.B.; GUIMARÃES, M.P. Gastrointestinal parasites of sheep, municipality of Lajes, Rio Grande do Norte, Brazil. Revista Brasileira de Parasitologia Veterinária, v. 21, n. 1, p. 71-73, 2012.

TEMBUE, A.A.S.M.; RAMOS, R.A.N.; LIMA, M.M.; FAUSTINO, M.A.G.; MEUNIER, I.M.J.; ALVES, L.C. Espécies do gênero Eimeria schneider, 1875 (Apicomplexa: Eimeriidae) em pequenos ruminantes, provenientes do município de Ibimirim, estado de Pernambuco. Veterinária notícias, v. 15, n. 2, p. 51-56, 2009.

VERCRUYSSE, J. The coccidia of sheep and goats in Senegal. Veterinary Parasitology. v. 10, n. 4, p. 297-306, 1982.

VIEIRA, L.S.; CAVALCANTE, A,C.R.; XIMENES, L.J.F. Evolution of infection with Eimeria species in hair sheep reared in Sobral, Ceará State, Brazil. Revue de Médicine Vétérinaire, v. 150, n. 6, p. 547-550, 1999.

WANG, C.R. Prevalence of coccidial infection in sheep and goats in northeastern China. Veterinary Parasitology, v. 174, p. 213-217, 2010.

YAKHCHALI, M.; REZAEI, A.A. The prevalence and intensity of Eimeria spp. infection in sheep of Malayer suburb, Iran. Archives of Razi Institute, v. 65, n. 1, p. 27-32, 2010. 